\title{
Simultaneous Dual Channel Phase Regeneration in SOAs
}

\author{
S. Sygletos, M. J. Power, F. C. Garcia Gunning, R. P. Webb, R. J. Manning, A. D. Ellis \\ Photonic Systems Group, Tyndall National Institute and Physics Department of University College \\ Cork, Lee Maltings, Cork, Ireland \\ stylianos.sygletos@tyndall.ie
}

\begin{abstract}
For the first time we demonstrate simultaneous suppression of phase distortion on two independent 10.7 Gbit/s DPSK modulated signal wavelengths using semiconductor optical amplifiers, realizing a compact phase sensitive amplifier with low power consumption.
\end{abstract}

\section{Introduction}

All optical regeneration is a promising approach for mitigating transmission impairments in high speed optical networks. However, in order to offer significantly reduced energy consumption when compared to optoelectronic based approaches in addition to low pump powers, solutions that may simultaneously process multiple phase modulated channels are required. Furthermore, integrated solutions are required for reduced manufacturing costs.

Multi-wavelength regeneration has been demonstrated primarily for OOK signal formats, primarily using schemes based on highly nonlinear fibre and optical filtering [1]-[2]. However, future long-haul networks will use of phase encoded signal formats, such as differential phase shift keying (DPSK), due to their reduced required optical signal to noise ratio (OSNR) requirements. All optical regenerators should provide suppression of the accumulated distortion, not only in the amplitude but also in the phase of the propagated signal. Initial schemes for phase modulated signals, focused on phase preserving amplitude regeneration or on a phase-to-amplitude format conversion [3]-[5]. Recently, phase sensitive amplification schemes have demonstrated remarkable capabilities for direct removal of the amplitude and phase distortion on a propagated signal [6]-[7] and preliminary work has demonstrated wavelength multiplexing capability using Brillouin suppressed highly nonlinear fibres [8]. However this result required pump launch powers in the region of 0.3 Watts per carrier (1 Watt total launch power) and the on-off phase sensitive gain remained constrained by stimulated Brillouin scattering.

In this paper we experimentally demonstrate, for the first time to our knowledge, simultaneous phase regeneration on two DPSK signals using a semiconductor optical amplifier (SOA) based phase sensitive amplifier (PSA) [9]. The PSA had $20 \mathrm{~dB}$ on-off gain contrast, was constructed as a "black-box" subsystem based on multipump degenerate four-wave mixing (FWM) and can be used as an independent in-line module in transmission links. Our scheme offers independent processing of each signal wavelength using a single nonlinear medium, suitable for integration into a single chip of hybrid integrated regeneration device. Performance evaluation with periodic input phase distortion showed improvement in terms of receiver sensitivity by more than $4 \mathrm{~dB}$, for both channels with required total launch power into the PSA SOA of less than $5 \mathrm{~mW}$.

\section{Experimental Setup and Results}

The experimental implementation of the proposed scheme is illustrated in Fig.1. At the transmitter, two separate laser sources emitting at $\lambda_{1}=1553 \mathrm{~nm}(\mathrm{ChA})$ and $\lambda_{2}=1553.4 \mathrm{~nm}(\mathrm{ChB})$ were used and modulated independently by pseudorandom binary sequences (PRBS) of $2^{31}$ 1 pattern length to give DPSK at the rate of 10.7 Gbit/s. A phase modulator, driven by a $3.3 \mathrm{GHz}$ sinusoidal wave from an independent RF source, was also used to introduce the corresponding phase distortion on both channels for evaluating the regenerative capabilities of the scheme. Subsequently, an Erbium doped fiber amplifier (EDFA) amplified the signals to a total power of $14 \mathrm{dBm}$ before launching them to the regenerator.

The proposed scheme is based on the phase sensitive interaction of the incoming signals with the local pumps of the subsystem. The scheme follows a "black-box" concept; and therefore it involves a phase synchronization stage where the processes of carrier extraction and phase locking takes place [10]. In the specific setup we made use of a free running continuous wave laser combined with the signal and injected into a single bulk SOA device to achieve simultaneous carrier extraction of the two incoming DPSK signals. The same technique has been implemented with a highly nonlinear fiber in [8]. The SOA was a polarization insensitive device with a length of $1 \mathrm{~mm}$, and presented a small signal gain of $24 \mathrm{~dB}$ and a saturated output power of $12 \mathrm{dBm}$. Contrary to the previous fiber based realizations, which necessitated optical power levels in the order of $\sim 1 \mathrm{~W}$, the same process is enabled here with 


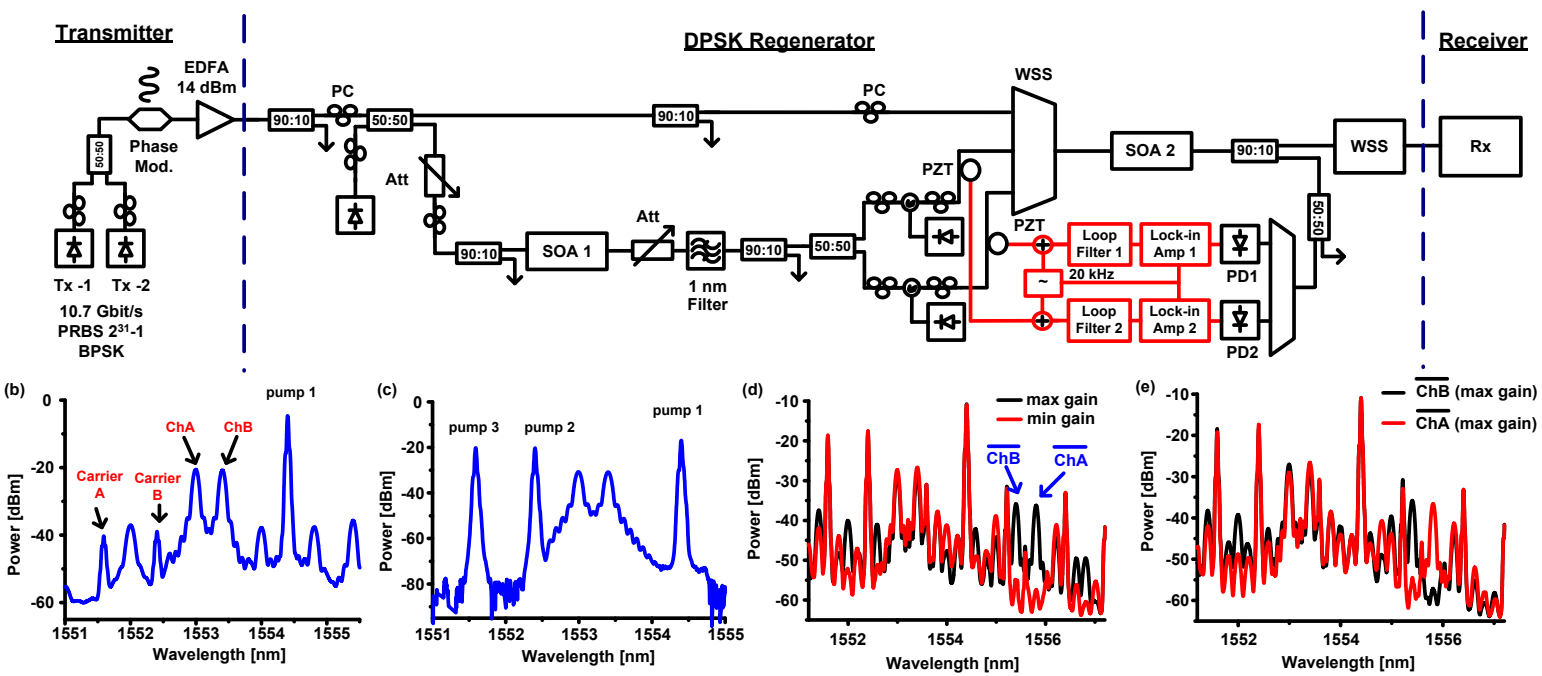

Fig. 1: (a) Experimental setup of the compact two channel PSA based on two SOA devices. (b) Optical spectrum after the carrier extraction stage at the output of SOA 1. (c) Optical spectrum at the output of the WSS. (d) Optical spectrum at the output of SOA-2 when both channels are locked either at the maximum and minimum phase sensitive gain state of the PSA. (e) Optical spectrum at the output of the SOA 2 when the channels are locked at different phase sensitive gain states of the PSA.

less than $4 \mathrm{~mW}$ total power at the input of the device. In addition, the compact size of the device allows carrier extraction parallel to the signal path without excessive thermally induced phase variation, thus avoiding excessive signal degradations while maintaining a robust PSA implementation. The corresponding optical spectrum taken at the output facet of the first SOA is depicted in Fig. 1(b). Subsequently, the generated carriers were selected by an optical filter to optically injection lock the two local pump lasers. As in previous implementations [7]-[8], the input power level to the injection locked lasers used to regenerate the quality of the $\mathrm{cw}$ signals was kept below $-35 \mathrm{dBm}$ per carrier, where any residual phase modulation of the input signal could be effectively suppressed [12]. A wavelength selective switch (WSS) combined the two incoming DPSK signals and the three local pumps, and subsequently directed them into the regeneration stage of the PSA. Fig 1(c) depicts the corresponding optical spectrum at the output of the WSS.

A second SOA was used at the regeneration stage of the PSA to enable the parametric interaction of the five input waves. This SOA was also $1 \mathrm{~mm}$ long but had a small signal gain of $32 \mathrm{~dB}$ and a saturated output power of 12 $\mathrm{dBm}$. The WSS was used to optimize the relative power levels of the five signals, resulting in a total launch power of less than $5 \mathrm{~mW}$. Through degenerate four-wave mixing, the two DPSK signals (ChA) and (ChB) interacted with their corresponding pump waves in the second SOA creating degenerate idlers that beat with them. However, contrary to highly nonlinear fibres, this beating mechanism in SOAs does not guarantee adequate phase squeezing as it is also affected by the non-parametric gain of the saturated device. This saturated gain benefits disproportionally the input signal over the generated idler reducing the contrast ratio. On the other hand, the idlers produced by nondegenerate FWM are not degraded by this competing gain process and so presented a significant phase sensitive gain and providing an efficient phase squeezing capability for these wavelength converted signals. In our case we considered the idler waves that were created from ChA and ChB symmetrically to the common pump (pump1) at the respective wavelengths of $\lambda_{4}=1555.81 \mathrm{~nm}(\overline{\mathrm{ChA}})$ and $\lambda_{3}$ $=1555.41 \mathrm{~nm}$ for $(\overline{\mathrm{ChB}})$. With an additional spectral inversion stage, the two output channels can be trivially brought back to the wavelength locations of the original signals. Fig. 1 (d) shows the optical spectrums at the output of the second SOA when the PSA was simultaneously locked at the maximum (black) or minimum (red) phase sensitive gain for both channels. Independent gain contrast ratios of $\sim 20 \mathrm{~dB}$ were achieved for both channels. Fig. 1(e) depicts the output spectra when the two channels were locked at two different phase sensitive gain states. The slow phase drifts of the interferometric setup were compensated by a feedback circuit which controlled two different piezoelectric fibre stretchers (PZTs), one for each pump path [8]. The stability of the system was greatly enhanced with respect to all fibre versions [7][8][10] due to the omission of long lengths of highly nonlinear fibre in the carrier extraction/phase locking path and the elimination of any fibre amplifiers from the black box regenerator. The use of a $2^{\text {nd }}$ WSS allowed us to select the regenerated channels. Finally at 
(a)

ChA / $\overline{\operatorname{ChA}}$

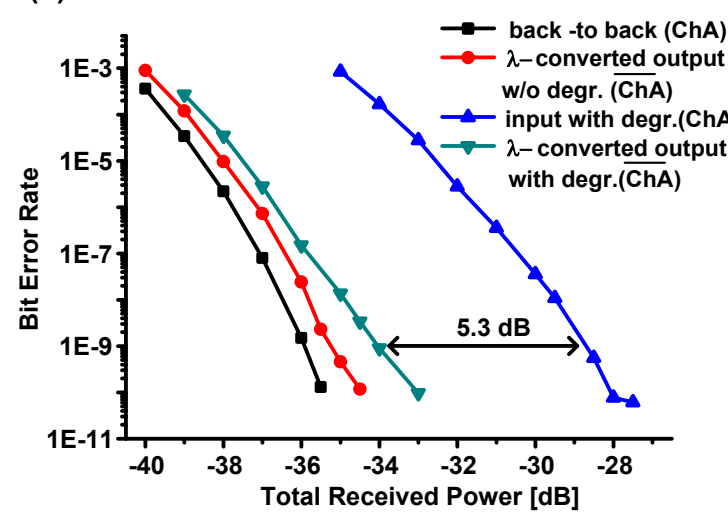

(c)
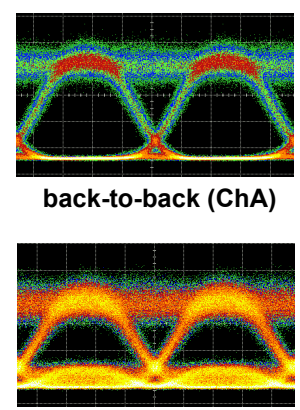

Input with degr. (ChA)

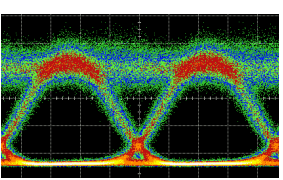

$\lambda$ - converted output w/o degr. ( $\overline{\mathrm{ChA}})$

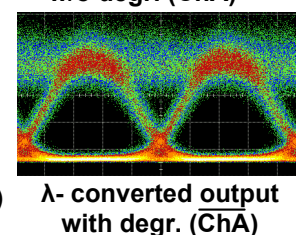

(b)

$\mathrm{ChB} / \overline{\mathrm{ChB}}$

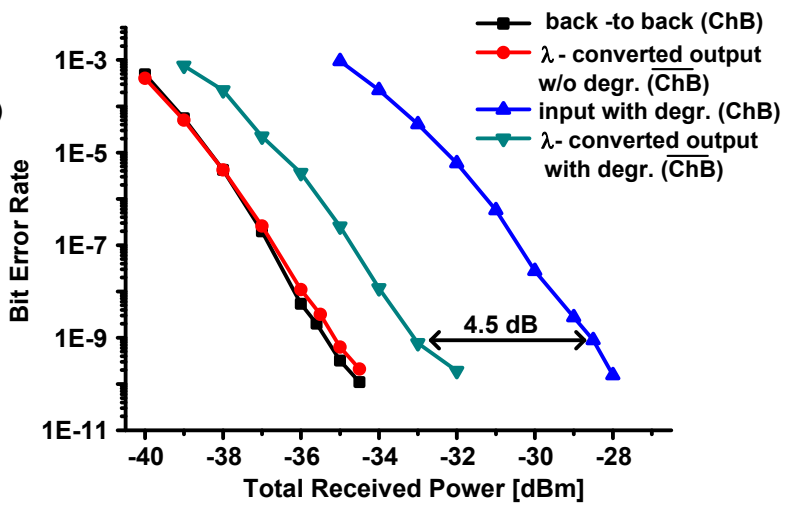

(d)

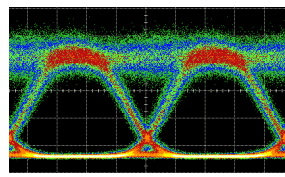

back-to-back (ChB)

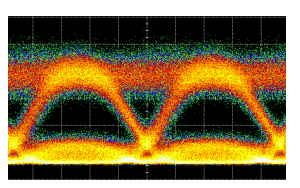

Input with degr. (ChB)

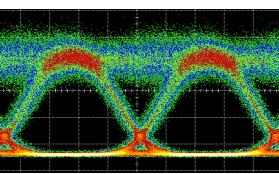

$\lambda$ - converted output w/o degr. ( $\overline{\mathrm{ChB}})$

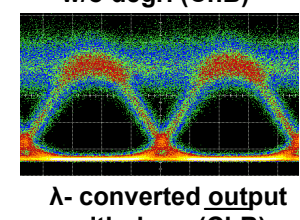

with degr. (ChB)

Fig. 2: BER measurements versus total received power (a) for $\mathrm{ChA}$ and the converted $\overline{\mathrm{ChA}}$ and (b) for $\mathrm{ChB}$ and the converted $\overline{\mathrm{ChB}}$ at input/output of PSA with/without the presence of input periodic degradation. Corresponding eye diagrams (c) for $\mathrm{ChA} / \overline{\mathrm{ChA}}$ and (d) for $\mathrm{ChB} / \overline{\mathrm{ChB}}$

the receiver, 1-bit delay $A M Z I$ was introduced to demodulate the signal back to NRZ prior to error detection using a $50 \mathrm{GHz}$ photodetector

The performance of our proposed scheme was assessed in terms of eye diagrams and measured bit-error-rates (BER) after single ended differential detection. Selections of the results are shown in Fig. 2 (a-d). The BER curves were been taken as a function of the total received power. Without input distortion errorfree operation for the two converted signals $(\overline{\mathrm{ChA}}$ and $\overline{\mathrm{ChB}})$ was achieved with receiver sensitivity penalties, at BER of $10^{-9}$, of less than $1 \mathrm{~dB}$. A phase distortion was introduced which reduced the receiver sensitivity by $7.14 \mathrm{~dB}$ for $\mathrm{ChA}$ and by $6.7 \mathrm{~dB}$ for $\mathrm{ChB}$ and degraded severely the respective eye diagrams. In that case, the PSA demonstrated the anticipated phase squeezing capability by improving the receiver sensitivities of the converted signals (5.3dB for $\overline{\mathrm{ChA}}$ and by $4.5 \mathrm{~dB}$ for $\overline{\mathrm{ChB}}$ ) and restoring the corresponding eye diagrams. All eye diagrams were taken at a power level of $28 \mathrm{dBm}$ with the same accumulation period.

\section{Conclusions}

We have proposed and experimentally implemented, for the first time to our knowledge, a compact, in-line PSA using only passive components and semiconductor optical amplifiers which allowed multichannel regeneration of phase encoded signals at $\sim \mathrm{mW}$ level powers. The regenerative performance of our scheme has been evaluated against periodic phase distortion for two independent 10.7Gbit/s DPSK signals. Both channels demonstrated significant restoration of the signal quality, translating to more than $\sim 4.5 \mathrm{~dB}$ receiver sensitivity improvement.

The work has been supported by Science Foundation Ireland under the grant number 06/IN/I969.

\section{References}

[1] L. Provost et al, in Proc OFC 2009, OWD7

[2] S. Boscolo et al., in Proc. ECOC 2002, P3.12

[3] K. Cvecek et al, PTL 19, 1475-1477, 2007

[4] M Matsumoto et al," Optics Express 16, 11169, (2008)

[5] I.Kang et al., in Proc. ECOC 2005, Thu 4.3.3

[6] K. Croussore, G. Li, IEEE JSTQE 14, 648-658 (2008).

[7] R. Slavík, et al., Nature Photonics 4, 690-695 (2010).

[8] S.Sygletos et al., Optics Express 19, B938-B945 (2011)

[9] R. P. Webb et al., Optics Express 19, 20015 (2011)

[10] S. Sygletos et al., in Proc ICTON (2010)

[11] R. Weerasuriya et al. in Proc. OFC 2010, OWT6.

[12] E. K. Lau, et al., JLT 26, 2584-2593, (2008). 